\title{
Photoproduction of hidden-bottom pentaquark and related topics
}

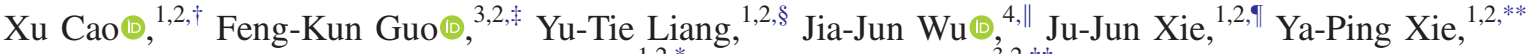 \\ Zhi Yang, ${ }^{1,2, *}$ and Bing-Song Zou ${ }^{3,2, \dagger \dagger}$ \\ ${ }^{1}$ Institute of Modern Physics, Chinese Academy of Sciences, Lanzhou 730000, China \\ ${ }^{2}$ School of Nuclear Science and Technology, University of Chinese Academy of Sciences, \\ Beijing 100049, China \\ ${ }^{3}$ CAS Key Laboratory of Theoretical Physics, Institute of Theoretical Physics, \\ Chinese Academy of Sciences, Beijing 100190, China \\ ${ }^{4}$ School of Physical Sciences, University of Chinese Academy of Sciences, Beijing 100049, China
}

(Received 20 January 2020; accepted 19 March 2020; published 10 April 2020)

\begin{abstract}
Because of the discovery of the hidden-charm pentaquark $P_{c}$ states by the LHCb Collaboration, the interests in the candidates of hidden-bottom pentaquark $P_{b}$ states are increasing. They are anticipated to exist as the analogs of the $P_{c}$ states in the bottom sector and predicted by many models. We give an exploration of searching for a typical $P_{b}$ in the $\gamma p \rightarrow \Upsilon p$ reaction, which shows a promising potential to observe it at an electron-ion collider. The possibility of searching for $P_{b}$ in open-bottom channels is also briefly discussed. Meanwhile, the $t$-channel nonresonant contribution, which in fact covers several interesting topics at low energies, is systematically investigated.
\end{abstract}

DOI: 10.1103/PhysRevD.101.074010

\section{INTRODUCTION}

Since the discovery of the $X(3872)$ by the Belle Collaboration [1], a rich spectrum of exotic states has been emerging; see comprehensive reviews in Refs. [2-9]. They not only shed new insight into the study of the hadron spectrum and structure but also deepen our understanding of nonperturbative properties of QCD. Among these states, the charged $Z_{c}(3900)$ and $Z_{c}(4020)$ found, respectively, in the $J / \psi \pi^{ \pm}[10,11]$ and $h_{c} \pi^{ \pm}$[12] systems seem to be surely exotic since they must contain at least one additional light quark and antiquark pair besides the hidden pair of $c \bar{c}$ to match the electric charge. Their partners in the bottomonium sector, namely, the $Z_{b}(10610)$ and $Z_{b}(10650)$, were firmly established by Belle in several different decay modes [13]. The spin and parity of these states are determined unambiguously to be $1^{+}$by the amplitude analysis of

\footnotetext{
* Corresponding author. zhiyang@impcas.ac.cn

caoxu@impcas.ac.cn

fkguo@itp.ac.cn

\$liangyt@impcas.ac.cn

"wujiajun@ucas.ac.cn

Txiejujun@impcas.ac.cn

**xieyaping@impcas.ac.cn

zoubs@itp.ac.cn
}

Published by the American Physical Society under the terms of the Creative Commons Attribution 4.0 International license. Further distribution of this work must maintain attribution to the author(s) and the published article's title, journal citation, and DOI. Funded by SCOAP.
BESIII [14] and Belle [15], except for the $Z_{c}$ (4020), which is believed to be of the same quantum numbers by most of the models. Their masses are very close to the $S$-wave thresholds of the corresponding open-flavor channels $D \bar{D}^{(*)}$ and $B \bar{B}^{(*)}$, respectively. As for their strange partner $Z_{s}$, so far, the BESIII Collaboration has not found a signal in the $\phi \pi$ spectrum of $e^{+} e^{-} \rightarrow \phi \pi \pi$ [16].

In the baryon sector, the hidden-strangeness pentaquark $P_{s}$ states containing only light quarks are expected in constituent quark models $[17,18]$ and in models considering the QCD van der Waals force $[19,20]$. But they are not explicitly found at present after long searching for them in $\pi N$ and $\gamma N$ reactions [21]. Other reactions and decays were suggested to study them from the theoretical side [22-28]. Interestingly, no narrow peaks were found in the total cross section of near-threshold $\gamma p \rightarrow \phi p$, but a nonmonotonic structure, found in the differential cross section by LEPS Collaboration [29], would imply a very wide (around $500 \mathrm{MeV}$ ) state [30,31]. There is also no evident signal in the $\phi p$ energy spectrum of the process $\Lambda_{c}^{+} \rightarrow \phi p \pi^{0}$ [32], which was shown in Ref. [33] to be not a good choice for the search of $P_{s}$ due to the presence of triangle singularities (for a recent review, see Ref. [9]) and the tiny phase space. However, in the charm sector, the astonishing observation of $P_{c}$ states by the LHCb Collaboration [34,35] has provided us an insightful place to study the exotic baryons in the charm sector, the existence of which were anticipated by several models [36-39]. The photoproduction reactions of these states with two-body final states, first proposed in Ref. [40] and followed by other works [41,42], are an 

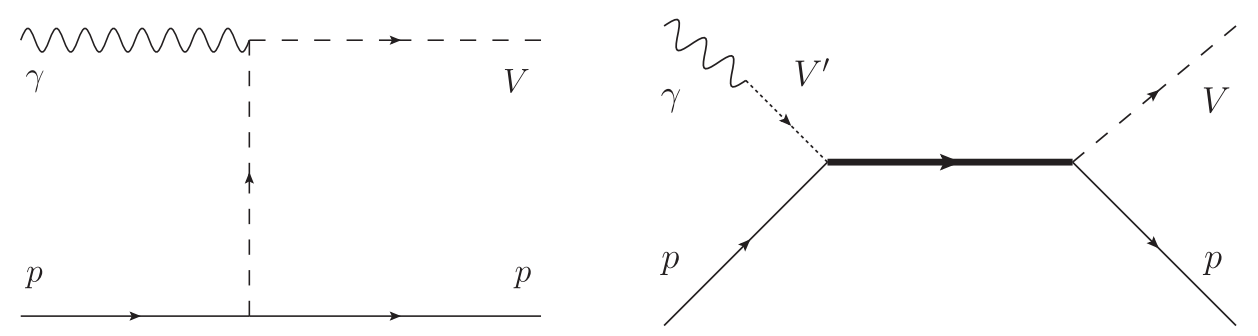

FIG. 1. Feynman diagrams for $\gamma p \rightarrow \Upsilon p$. Left: the $t$-channel contribution with the Pomeron or two-gluon exchange. Right: the $P_{b}$ production in the $s$ channel. $V$ labels the $\Upsilon$ meson. $V^{\prime}$ denotes the possible vector meson, including $\rho, \omega, \phi$, and $\Upsilon$, in the vector-meson dominance model.

exceptional platform to exclude their nonresonant possibility, because the on-shell conditions required by the triangle singularities discussed in Refs. [43-47] cannot be satisfied. The upper limit of the $P_{c}$ photoproduction cross section in $\gamma p \rightarrow J / \psi p$ was determined recently by the GlueX Collaboration [48], constraining the branching ratios of the $P_{c}$ decays into the $J / \psi p$ mode together with the results at $\mathrm{LHCb}$ [49]. Because of the null results in the GlueX data, double polarization observables were proposed to be a benchmark in the search of pentaquark photoproduction [50]. Although the nature of these exotic states is under discussion [51-57], they motivated the speculation from heavy-quark spin symmetry that there should be seven molecular pentaquarks in two spin multiplets [58-60]. Motivated by the heavy quark flavor symmetry for the potential between heavy mesons and baryons, the correspondence of these states in the bottom sector, labeled as $P_{b}$ here, are expected to be surely existing [42,61-63]. Unlike the $P_{c}$, they cannot be produced through the decay of heavier baryons. Therefore, they can only be directly produced in high-energy processes, such as the ep, $\gamma p$ scattering and the $p p$ collisions.

In this paper, we will discuss the possibilities of searching for one of typical $P_{b}$ states, the bottom analogs of $P_{c}$, in the photoproduction of the bottomonium channel $\gamma p \rightarrow \Upsilon p$ at electron-ion colliders (EICs). To this end, we first explore the nonresonant contribution to the $\gamma^{*} p \rightarrow \Upsilon p$ in Sec. II. This is very meaningful on its own right because several subjects are relevant to it. The detailed investigation of the $P_{b}$ contribution is presented in Sec. III. At last, we finish with a short summary in Sec. IV.

\section{NONRESONANT CONTRIBUTION}

The main purpose of studying the photo- and electroproduction of vector heavy quarkonia off the nucleon is to study the gluon component within the nucleon probed by heavy quarks. The low energies are also important for several other topics which are critically relevant. First, the near-threshold region would provide a clue for the quarkonium-nucleon interaction. The measured cross sections have been used to extract the $J / \psi p$ scattering length [64,65], whereas the $\Upsilon p$ scattering length is rarely known due to the lack of data. Second, it was proposed to be a promising platform to probe the trace anomaly term in the QCD energy-momentum tensor and the proton mass decomposition, resulting in a deep exploration of the origin of the nucleon mass $[66,67]$.

Since the discovery of the $J / \psi$, its photoproduction has attracted plenty of interest from both the experimental and theoretical aspects. Because the bottom quark is heavier than the charm one, the $\Upsilon$ photoproduction has its own merits. The multipole expansion $[66,68]$ converges more quickly. Relative uncertainties of the current quark mass and the running coupling constant are much smaller. This is an essential advantage for the theoretical calculation because the amplitudes are expected to be proportional to powers of these quantities. Last but not at least, it is ideal to search for hidden-bottom pentaquark candidates. The GlueX Collaboration has searched for the $P_{c}$ in the near-threshold region of the $\gamma p \rightarrow J / \psi p$ [48] as mentioned above. The $P_{b}$ states, whose lowest mass in many theoretical models is expected to be lying above $\Upsilon_{p}$ production threshold, making $\gamma p \rightarrow \Upsilon p$ as a perfect place to hunt for them. However, the data of the $\Upsilon$ production below $100 \mathrm{GeV}$ have never been measured up to now, and it becomes one of the main concerns of the proposed Electron-Ion Collider in China (EicC), as proposed in Ref. [69].

To explore the possibility of studying the $\Upsilon$ production at relative low energies, we need to estimate its cross section as a premise, with the help of a reliable model to extrapolate from high to low energies. The nonresonant contribution would come from the $t$-channel two-gluon or Pomeron exchange, as shown in Fig. 1. A rough evaluation of total cross section reads as

$$
\sigma\left(\gamma^{*} p \rightarrow V p\right)=\mathcal{N} W^{\delta\left(Q^{2}\right)}=\mathcal{N} W^{\alpha+\beta \ln \left(Q^{2}+M_{V}^{2}\right)},
$$

which is suggested by the empirical formula of the deeply virtual meson production (DVMP) $\gamma^{*} p \rightarrow V p$ [70]. Here, $M_{V}$ is the $\Upsilon$ mass, $W$ is the $\gamma p$ c.m. energy, and $Q^{2}$ is the photon virtuality. The advantage of this simple parametrization is that it is applicable to all DVMP processes with proper $Q^{2}$ dependence. The parameters $\alpha$ and $\beta$ have been determined by the DVMP data to be $\alpha=0.31 \pm 0.02$ and $\beta=0.13 \pm 0.01$ by Favart, Guidal, Horn, and Kroll 


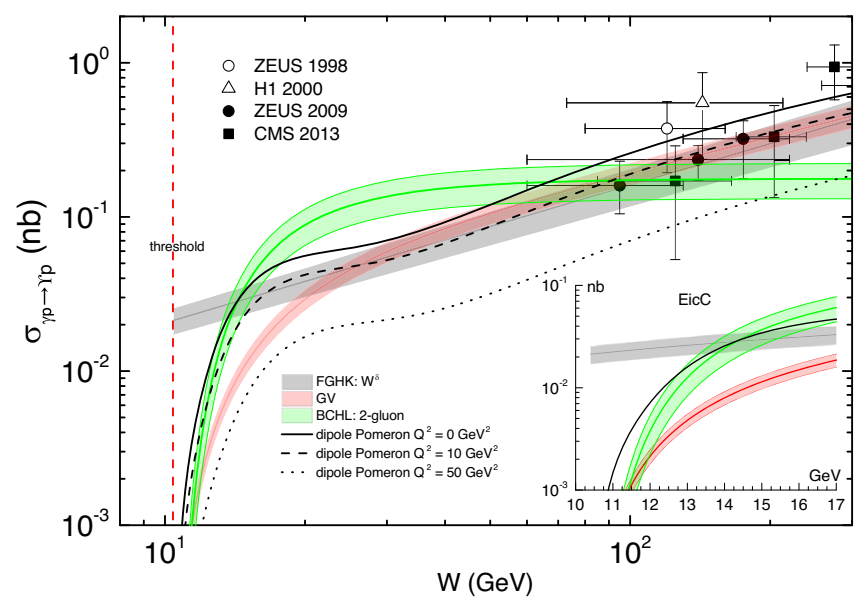

FIG. 2. The nonresonant contribution to the $\gamma^{*} p \rightarrow \Upsilon p$ as a function of the c.m. energy of $\gamma^{*} p, W$. The data are from ZEUS [75,76], H1 [77], and CMS [73]. The data above $300 \mathrm{GeV}$ from CMS [73] and LHCb [74] were used in the fit in order to determine the overall normalization but are not shown. The models include the DVMP empirical formula (FGHK) [70], twogluon exchange model (BCHL) [78], the parametrization in Ref. [64] (GV), and the soft dipole Pomeron [79,80]. The cross sections of $\gamma^{*} p \rightarrow \Upsilon p$ under $Q^{2}=10$ (dashed line) and $50 \mathrm{GeV}^{2}$ (dotted line) are also given by the soft dipole Pomeron model. The inserted subplot on the right bottom enlarges the energy region covered by the proposed EicC.

(FGHK) [70]. Correspondingly, $\delta\left(Q^{2}=0\right)=0.89 \pm 0.05$, confronted with the perturbative QCD prediction $\delta \sim 1.7$ [71] and ZEUS results $\delta=0.69 \pm 0.02 \pm 0.03$ [72]. The normalization $\mathcal{N}$ is determined by the data of $\gamma p \rightarrow \Upsilon p$ at high energies to be $2.62 \pm 0.38$, where the experimental uncertainty of $W$ is not taken into account. The result is shown as the gray band in Fig. 2, together with those from a few other models. As can be seen, the general trend of highenergy data with large errors follows the exponential behavior in Eq. (1). Note that the data above $300 \mathrm{GeV}$ up to $2 \mathrm{TeV}$ from CMS [73] and LHCb [74] were used in the fit in order to determine the overall normalization; they also follow the exponential behavior though not shown in the figure.

Gryniuk and Vanderhaeghen $(\mathrm{GV})$ adopted the parametrization for the cross section [64]

$\sigma(\gamma p \rightarrow V p)=\left(\frac{e f_{V}}{M_{V}}\right)^{2} \frac{\mathcal{N}}{2 W q_{\gamma p}}\left(\frac{q_{V p}}{q_{\gamma p}}\right)\left(1-\frac{\nu_{\mathrm{el}}}{\nu}\right)^{b_{\mathrm{el}}}\left(\frac{\nu}{\nu_{\mathrm{el}}}\right)^{a_{\mathrm{el}}}$,

where $f_{V}$ is the vector meson decay constant, $\nu=$ $\left(W^{2}-m_{p}^{2}-M_{V}^{2}\right) / 2$, and $\nu_{\mathrm{el}}=m_{p} M_{V}$. The quantities $q_{\gamma p}$ and $q_{V p}$ denote the magnitude of the 3-momenta in the c.m. frame of initial and final states, respectively. The parameters $a_{\mathrm{el}}=1.27 \pm 0.17$ and $b_{\mathrm{el}}=1.39 \pm 0.01$ are determined by the data of $\gamma p \rightarrow J / \psi p$. Good agreement is found after fitting the normalization $\mathcal{N}=0.014 \pm 0.002$ to the high-energy data, as shown by the red band in Fig. 2. This simple parametrization generally preserves the exponential trend at high energies and surprisingly agrees very well with the data above $100 \mathrm{GeV}$.

The two-gluon exchange model proposed by Brodsky, Chudakov, Hoyer, and Laget (BCHL) suggests the $t$ dependent cross sections [78]

$$
\frac{\mathrm{d} \sigma_{2 g}}{\mathrm{~d} t}(\gamma p \rightarrow V p)=\mathcal{N}_{2 g} \frac{(1-x)^{2}}{R^{2} M_{V}^{2}} \frac{1}{16 \pi} e^{b t},
$$

with $R=1 \mathrm{fm}, x=\left(2 m_{p} M_{V}+M_{V}^{2}\right) /\left(W^{2}-m_{p}^{2}\right)$, and the transfer-momentum squared $t$. We use the slope parameter $b=1.13 \mathrm{GeV}^{-2}$ in the original scheme, which is compatible with the measured one $b=1.25 \pm 0.20 \mathrm{GeV}^{-2}$ at $W=11 \mathrm{GeV}$ for the $J / \psi$ production [81]. The corresponding result is shown as the green band in Fig. 2. The normalization $\mathcal{N}_{2 g}$ is adjusted to the data around $100 \mathrm{GeV}$ because obviously this model cannot describe the data at high energies. The same authors also proposed the form of three-gluon exchange with an unknown normalization. It is premature to discuss such a contribution at present because of lack of data below $100 \mathrm{GeV}$.

Several Pomeron models have been constructed [82], but few of them have been used to study the case of the $\Upsilon$. The soft dipole Pomeron model, put forward by Martynov et al. $[79,80]$, preserves unitarity bounds with a double Regge pole with an intercept equal to 1. By fitting to all the available data of $\gamma^{*} p \rightarrow V p$ at that moment, the model predicts the behavior of $\gamma^{*} p \rightarrow \Upsilon p$, which is consistent with the measured data afterward; see the black curves in Fig. 2. Besides the usual exponential tendency at high energies, additional small fluctuations are observed. The shoulder shape around $20 \mathrm{GeV}$ is caused by a Regge pole mainly contributing to low energies. The trough around $30 \mathrm{GeV}$ is from the interference between two Regge poles. We also show the $Q^{2}$ dependence of the cross sections, which tend to be more moderate with larger $Q^{2}$ as expected from the $\left(Q^{2}+M_{V}^{2}\right)^{-1}$ behavior. Sibirtsev et al. also concluded that two Regge trajectories were required to describe the data of $\gamma^{*} p \rightarrow J / \psi p$ over a wide energy range after comparing various models [83]. This is different from most of the Pomeron models with only one Regge trajectory $[40,84]$.

Figure 2 shows that various models can describe the data at high energies comparably well, except the two-gluon exchange model, which is designed to focus on the nearthreshold region. However, the inserted subfigure in Fig. 2 shows that the deviations between different models are large at low energies, which are covered by the proposed EicC. The empirical formula of DVMP, as a guideline and a rough upper limit, does not take into account the influence from phase space, which is significant at low energies as one can easily anticipate. The soft dipole Pomeron model 
TABLE I. Parameters of $P_{b}$ in models. Here, we only list the $P_{b}$ with the mass around $11.12 \mathrm{GeV}$, and the other $P_{b}$ is not included.

\begin{tabular}{|c|c|c|c|c|}
\hline$P_{b}$ & Mass $M(\mathrm{GeV})$ & Width $\Gamma(\mathrm{MeV})$ & $\Gamma\left(P_{b} \rightarrow \Upsilon p\right)$ & $\mathcal{B}\left(P_{b} \rightarrow \Upsilon p\right)$ \\
\hline Wu et al. $[61]$ & 11.10 & 1.33 & 0.51 & 0.38 \\
\hline Karliner and Rosner $[42,63]$ & 11.14 & $39^{\mathrm{c}}$ or $61^{\mathrm{b}}$ & $\ldots$ & 0.1 \\
\hline Huang et al. $[85,86]$ & $11.09-11.14^{\mathrm{a}}$ & 7.0 & 4.4 & 0.63 \\
\hline Lin et al. [87] & $\ldots$ & $30-300$ & $\cdots$ & $0.0003-0.0013$ \\
\hline Yang et al. [88] & 11.14 & $\ldots$ & $\ldots$ & $\ldots$ \\
\hline Xiao and Oset [62] & $10.96-11.022$ & $2-110$ & $\cdots$ & $\cdots$ \\
\hline Shen et al. [89] & 11.120 & 25 & $\ldots$ & $\cdots$ \\
\hline Gutsche and Lyubovitskij [90] & 11.125 & $\cdots$ & 3.27 & $\cdots$ \\
\hline Gutsche and Lyubovitskij [90] & 11.13 & $\cdots$ & 6.57 & $\cdots$ \\
\hline
\end{tabular}

${ }^{\mathrm{a}}$ If all closed channels are included, it is $10.304\left(1 / 2^{-}\right)$and $10.382\left(3 / 2^{-}\right)$.

${ }^{\mathrm{b}}$ Rough estimation from phase space ratio $\Gamma\left(P_{b}\right) / \Gamma\left(P_{c}\right)=k_{\text {out }}\left(P_{b}\right) / k_{\text {out }}\left(P_{c}\right)$.

${ }^{\mathrm{c}}$ Assume the same width with $P_{c}(4450)$ at LHCb.

overlaps with the two-gluon exchange one within uncertainties but is larger in the very close-to-threshold range. The GV parametrization is smaller than the other models below $20 \mathrm{GeV}$.

In a short conclusion, the soft dipole Pomeron model and the GV parametrization are both compatible with highenergy data and give the expected behavior of the phase space at low energies. So, they serve as a good input for the study of the nonresonant contribution to the ep $\rightarrow$ ep $\Upsilon$ process. Because the parameters in the soft dipole Pomeron model are from a global fit to all the data, in the next section, we will use it as the nonresonant contribution to $\gamma p \rightarrow \Upsilon p$. We also use the empirical formula from DVMP as a crude estimation of the upper limit of the nonresonant contribution. Besides, other models that are available to calculate the cross section of the $\gamma^{*} p \rightarrow J / \psi p$ can also be extended to the case of the $\gamma^{*} p \rightarrow \Upsilon p$. However, most of them have more undetermined parameters owing to lack of data of the $\Upsilon$ production, so we do not consider them at present.

\section{III. $P_{b}$ AS A RESONANCE IN PHOTOPRODUCTION}

We list the properties of a typical $P_{b}$ predicted by various phenomenological models which used the $P_{c}$ as inputs in Table I. We do not attempt to collect all the models here because of the still increasing literature. We would like to point out that nearly all models predict a resonant state with a mass around $11.12 \mathrm{GeV}$, which couples to the $\Upsilon p$ channel, while the total width differs due to detailed constructions of the models, ranging from 30 to $300 \mathrm{MeV}$. In this paper, we will adopt the mass of $11.12 \mathrm{GeV}$ with two possible width values 30 and $300 \mathrm{MeV}$. Later on, they are dubbed as the narrow $P_{b}$ and the wide $P_{b}$, respectively. The spin $J=1 / 2$ is used here as a representative choice. Other $P_{b}$ states with different quantum numbers can be similarly calculated since the production cross section is proportional to $2 J+1$ in our prescription.
The production cross section of the exotic $P_{b}$ in the reaction $\gamma p \rightarrow \Upsilon p$, as shown by the Feynman diagram in Fig. 1, can be written as

$\sigma_{P_{b}}=\frac{2 J+1}{\left(2 s_{1}+1\right)\left(2 s_{2}+1\right)} \frac{4 \pi}{k_{\mathrm{in}}^{2}} \frac{\Gamma^{2}}{4} \frac{\mathcal{B}\left(P_{b} \rightarrow \gamma p\right) \mathcal{B}\left(P_{b} \rightarrow \Upsilon p\right)}{(W-M)^{2}+\Gamma^{2} / 4}$.

Here, $k_{\text {in }}$ is the magnitude of the initial-state 3-momentum in the c.m. frame, and $s_{1}$ and $s_{2}$ are the spins of initial photon and proton, respectively. Because the mass $M$ of $P_{b}$ is very large, this formula is a very good approximation even for the wide $P_{b}$. If assuming that the $P_{b} \rightarrow$ $\gamma p$ is dominated by only the heavy vector meson in the vector meson dominance model, e.g., $V^{\prime}=\Upsilon$ in Fig. 1, the branching ratio $\mathcal{B}\left(P_{b} \rightarrow \gamma p\right)$ is proportional to $\mathcal{B}\left(P_{b} \rightarrow \Upsilon p\right)[41,42,49]$,

$$
\mathcal{B}\left(P_{b} \rightarrow \gamma p\right)=\frac{3 \Gamma\left(\Upsilon \rightarrow e^{+} e^{-}\right)}{\alpha M_{\Upsilon}} \frac{k_{\text {in }}}{k_{\text {out }}} \mathcal{B}\left(P_{b} \rightarrow \Upsilon p\right),
$$

which has assumed the lowest orbital excitation $L=0$ between the $\Upsilon$ and the proton. Here, $\alpha$ is the fine structure constant, $k_{\text {out }}$ is the magnitude of final-state 3-momentum in the c.m. frame, and the dilepton width $\Gamma\left(\Upsilon \rightarrow e^{+} e^{-}\right)=$ $1.34 \mathrm{keV}$ [91]. As a result, we have $\sigma_{P_{b}} \propto \mathcal{B}^{2}\left(P_{b} \rightarrow \Upsilon p\right)$. It shall be noted that the intermediate vector meson $V^{\prime}=\Upsilon$ in Fig. 1 is highly off shell, so a form factor would be present with a possible strong suppression, as pointed out in Ref. [92]. At present, the branching fraction $\mathcal{B}\left(P_{b} \rightarrow \gamma p\right)$ is not directly measured, so the magnitude of this form factor is unknown. As a result, $\mathcal{B}\left(P_{b} \rightarrow \gamma p\right)$ above needs to be understood as an effective branching ratio with this factor absorbed. Recently, the measurement of GlueX at Jefferson Lab (JLab) Hall-D has given the upper limit of $\mathcal{B}\left(P_{c}^{+} \rightarrow\right.$ $J / \psi p)$ to be several percent without considering this offshell factor. The $\mathrm{LHCb}$ results indicate a stringent lower limit of $\mathcal{B}\left(P_{c}^{+} \rightarrow J / \psi p\right)$ to be $0.05 \% \sim 0.5 \%$ [49]. We use these values of $P_{c}$ as a reference and adopt 

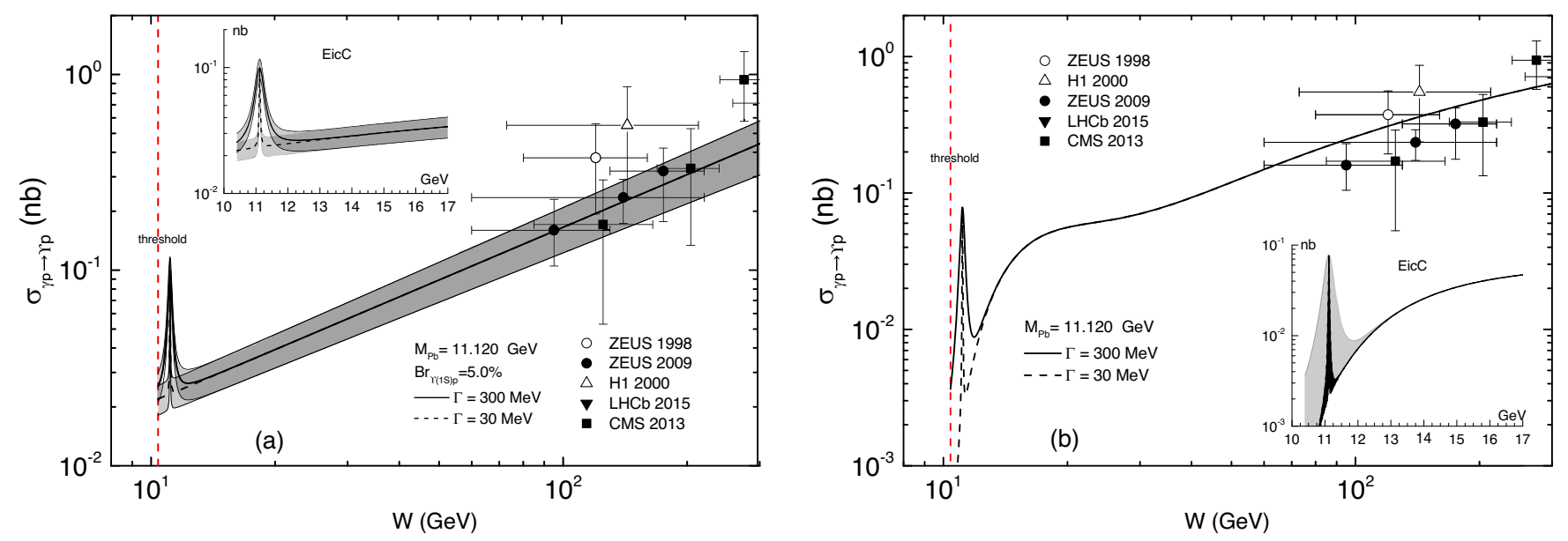

FIG. 3. The cross section of $\gamma p \rightarrow \Upsilon p$ with $\mathcal{B}\left(P_{b} \rightarrow \Upsilon p\right)=5 \%$ as a function of the c.m. energy. The data are the same as those in Fig. 2. The inserted subplots enlarge the energy region covered by the proposed EicC. (a) The nonresonant contribution of the DVMP empirical formula is used. (b) The nonresonant contribution of the soft dipole Pomeron model is used. The bands in the inserted subplot on right bottom represent the range of $0.5 \%<\mathcal{B}\left(P_{b} \rightarrow \Upsilon p\right)<5 \%$.

$0.5 \%<\mathcal{B}\left(P_{b} \rightarrow \Upsilon p\right)<5 \%$ for $P_{b}$. The calculated values in most of the models in Table I are within this chosen range, except one of them approaching to about $0.01 \%$ [87].

The nonresonant contribution studied in Sec. II is considered as the smooth background of $P_{b}$. The interference effect between them in the total and differential cross sections is not significant because the $t$-channel Pomeron exchange contributes only to the forward angles while the $s$-channel resonances are present in full angles. The calculation of $\gamma p \rightarrow J / \psi p$ confirms this expectation [92]. The hereafter error bands are from the uncertainty of nonresonant contribution but do not include the errors of the mass $M$ and width $\Gamma$ of $P_{b}$, just because it is too premature to consider them at this stage.

The calculated results are presented in Fig. 3(a) with $\mathcal{B}\left(P_{b} \rightarrow \Upsilon p\right)=5 \%$ and the nonresonant contribution of the DVMP empirical formula in Eq. (1). The background is smooth within the EicC energies in the range of 0.01$0.02 \mathrm{nb}$. The peak cross section of the narrow $P_{b}$ is around $0.1 \mathrm{nb}$ at most. The effects of both the narrow and wide $P_{b}$ are prominent, as can be seen. This is contrary to the decay of $\Lambda_{b}^{0} \rightarrow K^{-} J / \psi p$ at $\mathrm{LHCb}$, where a wide resonance is much harder to identify due to the more complicated background. Notice that the DVMP parametrization does not consider the phase space, so the results need to be considered as an upper limit in the low-energy region as already mentioned.

We show the results with the nonresonant contribution of the soft dipole Pomeron model in Fig. 3(b) with $0.5 \%<$ $\mathcal{B}\left(P_{b} \rightarrow \Upsilon p\right)<5 \%$. The background varies rapidly in the range of the EicC energies because of the phase space. The $P_{b}$ signal is still clearly visible if $\mathcal{B}\left(P_{b} \rightarrow \Upsilon p\right)>1.0 \%$. It would be difficult to find the $P_{b}$ with $\mathcal{B}\left(P_{b} \rightarrow \Upsilon p\right)$ as small as $0.5 \%$ in an unpolarized measurement, and therefore polarization observables are needed. The formalism for a detailed calculation of polarized measurements is well established $[21,40,92]$. But we will not pursue that aspect in this paper, because the interference is definitely essential but out of control due to lack of low-energy data. The $t$-dependence of the nonresonant contribution in the soft Pomeron model is very close to $e^{b t}$ with the same value for the slope $b$ in Eq. (3) because this slope is mainly driven by the data of the $J / \psi$ production in the soft dipole Pomeron model. It would be very interesting to look into the slope for the $\Upsilon$ once data are available in the future.

As shown in Fig. 3(a), the nonresonant $\Upsilon$ photon production at EicC energies is around $0.02 \mathrm{nb}$ at most, and a reduction factor of about 5 is introduced by the twobody phase space; see Fig. 3(b). The resonant $P_{b}$ photon production in the peak energy is around $0.1 \mathrm{nb}$. For reactions at electron ion colliders, a roughly 2 orders of magnitude smaller cross section is anticipated for the electroproduction compared to the above photon production. Take the EicC as an example; about $5 \times 10^{4}$ signal events of $e p \rightarrow e P_{b} \rightarrow$ $e \Upsilon p$ are expected with an integrated luminosity of $50 \mathrm{fb}^{-1}$. Even after considering the small leptonic decay branching fraction of the $\Upsilon$ and the detection efficiency, the observation of this channel is still optimistic at the EicC. The produced $P_{b}$ is not far away from the central rapidity region at the EicC energies, which is good for detection. A detailed simulation is under investigation and will be soon available for publication [69].

The US-EIC project covers ep c.m. energies of 30$140 \mathrm{GeV}$ (eRHIC), and its optimal energy is around $100 \mathrm{GeV}$ [93,94]. It covers larger $Q^{2}$ range, and its electroproduction cross section of $\Upsilon p$ should be several times larger than that of EicC due to the much larger c.m. energies [69]. Its designed luminosity is around 1 order of magnitude higher than that of EicC $\left(2 \sim 4 \times 10^{33}\right.$ at EicC 
vs $10^{34}$ or higher at US-EIC). As a result, for the same duration of running time, the events produced at the USEIC will be tens of times more than those at EicC. The final particles of the reaction of interest in EicC is within the middle rapidity range, while they are distributed in the large rapidity range at the US-EIC due to much higher c.m. energies, challenging the design of detector coverage for studying this spectroscopy issue. A detailed comparison of pentaquark electroproduction at EicC and other EICs with higher energies (US-EIC and the Large Hadron electron Collider) will be given in a forthcoming manuscript [95].

\section{CONCLUSIONS}

In this paper, we made a detailed exploration of the nonresonant contribution to the $\gamma p \rightarrow \Upsilon p$, with the aim to find a reasonable estimation of the production rate at relatively low energies, where no data are available up to now. An extrapolation from the energies of the LHC and HERA data to low energies by several models gives us a reasonable estimate of the cross section below $100 \mathrm{GeV}$. We emphasize that this nonresonant contribution to the $\gamma p \rightarrow \Upsilon p$ is related to several appealing topics. It may give access to the $\Upsilon_{p}$ scattering length, which is a key parameter for understanding of whether a bottomonium can be bound with the nucleon and light nuclei. Our results in Fig. 2 in fact can be used to roughly estimate the scattering length, as done for that of $J / \psi p$ [65]. It could be also decisive for extracting the information of the trace anomaly contribution to the nucleon mass, and so finally solve the problem of the proton mass decomposition [67]. We would like to further remark that the larger mass of the $\Upsilon$ than the $J / \psi$ could make it a better place for studying these issues because the relative uncertainties of current quark mass and running coupling constants are smaller at high energies [91]

$$
\frac{\Delta m_{Q}}{m_{Q}} \simeq\left\{\begin{array}{ll}
2.5 \% \\
1.0 \%
\end{array}, \quad \frac{\Delta \alpha_{s}}{\alpha_{s}} \simeq\left\{\begin{array}{ll}
7.8 \% & \text { for } J / \psi \\
3.7 \% & \text { for } \Upsilon
\end{array} .\right.\right.
$$

These issues may be notably clarified by measurements at the electron proton colliders.

After the study of the nonresonant contribution, we conducted a careful estimation of the production of $P_{b}$ in $\gamma p \rightarrow \Upsilon p$ under the assumption that the $P_{b}$ naturally inherits features from the $P_{c}$. If it is found in the photoand electroproduction in the future, the $P_{b}$ will be firmly established as a genuine resonant state because resonantlike structures from triangle singularities are inapplicable to this reaction. We estimated the production yield at EIC machines based on the calculated cross sections and found that if the $\mathcal{B}\left(P_{b} \rightarrow \Upsilon p\right)$ is larger than $1.0 \%$ the $P_{b}$ states should be observed at the EicC through the $\gamma p \rightarrow$ $\Upsilon p$ process. On the other hand, if $\mathcal{B}\left(P_{b} \rightarrow \Upsilon p\right)$ is smaller than $1.0 \%$ as predicted by Ref. [87], then the $P_{b}$ states need to be searched for in the dominant decay channels $B^{(*)} \Lambda_{b}$ final states, whose branching fractions were predicted to be 2 to 3 orders of magnitude larger in Ref. [87]. Then, from Eq. (4), the cross section of the $P_{b}$ photoproduction in the $B^{(*)} \Lambda_{b}$ channel given by

$$
\sigma_{P_{b} \rightarrow B^{(*)} \Lambda_{b}}=\frac{\mathcal{B}\left(P_{b} \rightarrow B^{(*)} \Lambda_{b}\right)}{\mathcal{B}\left(P_{b} \rightarrow \Upsilon p\right)} \sigma_{P_{b} \rightarrow \Upsilon p}
$$

assuming the same production mechanism should also be larger by the same scale. However, the cross section for the $\gamma p \rightarrow B^{(*)} \Lambda_{b}$ also receives a $t$-channel contribution from the exchange of a bottom meson. The calculation of such a contribution is highly model dependent because of the presence of the off-shell form factor. As a reference, the channels $\gamma p \rightarrow \bar{D}^{0} \Lambda_{c}^{+}$and $\gamma p \rightarrow$ $\bar{D}^{* 0} \Lambda_{c}^{+}$are both calculated using an effective Lagrangian model in Ref. [92], and the latter is also computed with similar approach in Ref. [96]. These models suggests the $t$-channel $D^{0}$ exchange to be dominant; however, the magnitude depends significantly on the cutoff in the form factor, ranging in several orders [96]. The similar reaction in the bottom sector is anticipated to receive an even larger uncertainty due to the larger virtuality of the exchanged bottom meson. Nevertheless, an estimation from the semi-inclusive $\gamma p \rightarrow b \bar{b} X$ would give a good guideline. Since the cross section of the semi-inclusive $\gamma p \rightarrow b \bar{b} X$ at high energies is found to be 2 orders of magnitude larger than that of the $\gamma p \rightarrow \Upsilon p$ by experiments [97,98] and the next-to-leading-order QCD calculation [99,100], the open bottom channels $\bar{B}^{(*)} \Lambda_{b}$ from nonresonant contribution are expected to have a larger cross section than that of $\Upsilon p$ channels. So, these $P_{b}$ states may be observed at EIC machines through the $\gamma p \rightarrow \bar{B}^{(*)} \Lambda_{b}$ reaction, if the detection efficiency of weak decay particles is promoted. In particular, almost all of the possible open bottom modes will have the $\bar{B} \Lambda_{b}$ in the final states. So, a real measurement anticipated at EIC machines will surely clarify the situation to a large extent.

In brief, future EIC machines can be used to search for the hidden-bottom pentaquark $P_{b}$ states, as the bottom partners of the $P_{c}$, in the $\gamma p \rightarrow \Upsilon p$ and open-bottom processes. New insights are expected in the physics of exotic hadrons. Also, the EicC can measure the cross section of $\gamma p \rightarrow \Upsilon p$ in relatively low energies, covering a variety of interesting physics aspects.

\section{ACKNOWLEDGMENTS}

Useful discussions with Evgenij Martynov and $\mathrm{Nu} \mathrm{Xu}$ are gratefully acknowledged. We thank all the authors of the EicC white paper, especially Chao-Hsi Chang, KuangTa Chao, Hongxia Huang, and Qiang Zhao, for enlightening suggestions. This work was supported in part by the 
National Natural Science Foundation of China (Grants No. 11405222, No. 11621131001, No. 11835015, No. 11947302, No. 11975278, No. 11735003, No. 1191101015, and No. 11961141012), by the Pioneer Hundred Talents Program of Chinese Academy of Sciences
(CAS), by the Key Research Program of CAS (Grant No. XDPB09), by the CAS Key Research Program of Frontier Sciences (Grant No. QYZDB-SSW-SYS013), and by the CAS Center for Excellence in Particle Physics.
[1] S. K. Choi et al. (Belle Collaboration), Phys. Rev. Lett. 91, 262001 (2003).

[2] H.-X. Chen, W. Chen, X. Liu, and S.-L. Zhu, Phys. Rep. 639, 1 (2016).

[3] F.-K. Guo, C. Hanhart, U.-G. Meißner, Q. Wang, Q. Zhao, and B.-S. Zou, Rev. Mod. Phys. 90, 015004 (2018).

[4] R. F. Lebed, R. E. Mitchell, and E. S. Swanson, Prog. Part. Nucl. Phys. 93, 143 (2017).

[5] A. Esposito, A. Pilloni, and A. D. Polosa, Phys. Rep. 668, 1 (2017).

[6] S. L. Olsen, T. Skwarnicki, and D. Zieminska, Rev. Mod. Phys. 90, 015003 (2018).

[7] Y.-R. Liu, H.-X. Chen, W. Chen, X. Liu, and S.-L. Zhu, Prog. Part. Nucl. Phys. 107, 237 (2019).

[8] N. Brambilla, S. Eidelman, C. Hanhart, A. Nefediev, C. P. Shen, C. E. Thomas, A. Vairo, and C. Z. Yuan, arXiv:1907. 07583.

[9] F.-K. Guo, X.-H. Liu, and S. Sakai, Prog. Part. Nucl. Phys. (2020), https://doi.org/10.1016/j.ppnp.2020.103757.

[10] M. Ablikim et al. (BESIII Collaboration), Phys. Rev. Lett. 110, 252001 (2013).

[11] Z. Q. Liu et al. (Belle Collaboration), Phys. Rev. Lett. 110, 252002 (2013); 111, 019901(E) (2013).

[12] M. Ablikim et al. (BESIII Collaboration), Phys. Rev. Lett. 111, 242001 (2013).

[13] A. Bondar et al. (Belle Collaboration), Phys. Rev. Lett. 108, 122001 (2012).

[14] M. Ablikim et al. (BESIII Collaboration), Phys. Rev. Lett. 119, 072001 (2017).

[15] A. Garmash et al. (Belle Collaboration), Phys. Rev. D 91, 072003 (2015).

[16] M. Ablikim et al. (BESIII Collaboration), Phys. Rev. D 99, 011101 (2019).

[17] H. Huang, X. Zhu, and J. Ping, Phys. Rev. D 97, 094019 (2018).

[18] X. Liu, H. Huang, and J. Ping, Phys. Rev. C 98, 055203 (2018).

[19] H. Gao, T. S. H. Lee, and V. Marinov, Phys. Rev. C 63, 022201 (2001).

[20] J. He, H. Huang, D.-Y. Chen, and X. Zhu, Phys. Rev. D 98, 094019 (2018).

[21] X. Cao and H. Lenske, Phys. Lett. B 772, 274 (2017).

[22] X. Cao, Chin. Phys. C 39, 041002 (2015).

[23] X. Cao and J. P. Dai, Phys. Rev. D 100, 054004 (2019).

[24] X. Cao and J.-J. Xie, Chin. Phys. C 40, 083103 (2016).

[25] R. F. Lebed, Phys. Rev. D 92, 114030 (2015).

[26] J. He, Phys. Rev. D 95, 074031 (2017).
[27] C.-S. An, J.-J. Xie, and G. Li, Phys. Rev. C 98, 045201 (2018).

[28] H. Gao, H. Huang, T. Liu, J. Ping, F. Wang, and Z. Zhao, Phys. Rev. C 95, 055202 (2017).

[29] T. Mibe et al. (LEPS Collaboration), Phys. Rev. Lett. 95, 182001 (2005).

[30] A. Kiswandhi, J.-J. Xie, and S.-N. Yang, Phys. Lett. B 691, 214 (2010).

[31] A. Kiswandhi and S.-N. Yang, Phys. Rev. C 86, 015203 (2012); 86, 019904(E) (2012).

[32] B. Pal et al. (Belle Collaboration), Phys. Rev. D 96, 051102 (2017).

[33] J.-J. Xie and F.-K. Guo, Phys. Lett. B 774, 108 (2017).

[34] R. Aaij et al. (LHCb Collaboration), Phys. Rev. Lett. 115, 072001 (2015).

[35] R. Aaij et al. (LHCb Collaboration), Phys. Rev. Lett. 122, 222001 (2019).

[36] J.-J. Wu, R. Molina, E. Oset, and B.-S. Zou, Phys. Rev. Lett. 105, 232001 (2010).

[37] J.-J. Wu, R. Molina, E. Oset, and B.-S. Zou, Phys. Rev. C 84, 015202 (2011).

[38] W.-L. Wang, F. Huang, Z.-Y. Zhang, and B.-S. Zou, Phys. Rev. C 84, 015203 (2011).

[39] Z.-C. Yang, Z.-F. Sun, J. He, X. Liu, and S.-L. Zhu, Chin. Phys. C 36, 6 (2012).

[40] Q. Wang, X.-H. Liu, and Q. Zhao, Phys. Rev. D 92, $034022(2015)$.

[41] V. Kubarovsky and M. B. Voloshin, Phys. Rev. D 92, 031502 (2015).

[42] M. Karliner and J. L. Rosner, Phys. Lett. B 752, 329 (2016).

[43] F.-K. Guo, U.-G. Meißner, W. Wang, and Z. Yang, Phys. Rev. D 92, 071502 (2015).

[44] X.-H. Liu, Q. Wang, and Q. Zhao, Phys. Lett. B 757, 231 (2016).

[45] F.-K. Guo, U.-G. Meißner, J. Nieves, and Z. Yang, Eur. Phys. J. A 52, 318 (2016).

[46] M. Bayar, F. Aceti, F.-K. Guo, and E. Oset, Phys. Rev. D 94, 074039 (2016).

[47] X. H. Liu, G. Li, J.-J. Xie, and Q. Zhao, Phys. Rev. D 100, 054006 (2019).

[48] A. Ali et al. (GlueX Collaboration), Phys. Rev. Lett. 123, 072001 (2019).

[49] X. Cao and J. p. Dai, Phys. Rev. D 100, 054033 (2019).

[50] D. Winney, C. Fanelli, A. Pilloni, A. N. Hiller Blin, C. Fernández-Ramírez, M. Albaladejo, V. Mathieu, V. I. 
Mokeev, and A. P. Szczepaniak (JPAC Collaboration), Phys. Rev. D 100, 034019 (2019).

[51] R. Chen, Z. F. Sun, X. Liu, and S. L. Zhu, Phys. Rev. D 100, 011502 (2019).

[52] F.-K. Guo, H.-J. Jing, U.-G. Meißner, and S. Sakai, Phys. Rev. D 99, 091501 (2019).

[53] Z.-H. Guo and J. A. Oller, Phys. Lett. B 793, 144 (2019).

[54] M. I. Eides, V. Y. Petrov, and M. V. Polyakov, arXiv:1904. 11616.

[55] Z. G. Wang, Int. J. Mod. Phys. A 35, 2050003 (2020).

[56] A. Ali, I. Ahmed, M. J. Aslam, A. Y. Parkhomenko, and A. Rehman, J. High Energy Phys. 10 (2019) 256.

[57] T. J. Burns and E. S. Swanson, Phys. Rev. D 100, 114033 (2019).

[58] M.-Z. Liu, Y.-W. Pan, F.-Z. Peng, M. Sánchez Sánchez, L.-S. Geng, A. Hosaka, and M. P. Valderrama, Phys. Rev. Lett. 122, 242001 (2019).

[59] C.-W. Xiao, J. Nieves, and E. Oset, Phys. Rev. D 100, 014021 (2019).

[60] M. L. Du, V. Baru, F. K. Guo, C. Hanhart, U. G. Meißner, J. A. Oller, and Q. Wang, Phys. Rev. Lett. 124, 072001 (2020).

[61] J.-J. Wu, L. Zhao, and B.-S. Zou, Phys. Lett. B 709, 70 (2012).

[62] C.-W. Xiao and E. Oset, Eur. Phys. J. A 49, 139 (2013).

[63] M. Karliner and J. L. Rosner, Phys. Rev. Lett. 115, 122001 (2015).

[64] O. Gryniuk and M. Vanderhaeghen, Phys. Rev. D 94, 074001 (2016).

[65] I. Strakovsky, D. Epifanov, and L. Pentchev, arXiv:1911. 12686.

[66] D. Kharzeev, Proc. Int. School Phys. Fermi 130, 105 (1996).

[67] Y. Hatta and D.-L. Yang, Phys. Rev. D 98, 074003 (2018).

[68] H. Fujii and D. Kharzeev, arXiv:hep-ph/9807383.

[69] X. Cao, L. Chang, N. Chang et al., Nucl. Tech. 43, 020001 (2020).

[70] L. Favart, M. Guidal, T. Horn, and P. Kroll, Eur. Phys. J. A 52, 158 (2016).

[71] L. L. Frankfurt, M. F. McDermott, and M. Strikman, J. High Energy Phys. 02 (1999) 002.

[72] S. Chekanov et al. (ZEUS Collaboration), Eur. Phys. J. C 24, 345 (2002).

[73] CMS Collaboration, CERN Report No. CMS-PAS-FSQ13-009, 2016.

[74] R. Aaij et al. (LHCb Collaboration), J. High Energy Phys. 09 (2015) 084.

[75] J. Breitweg et al. (ZEUS Collaboration), Phys. Lett. B 437, 432 (1998).

[76] S. Chekanov et al. (ZEUS Collaboration), Phys. Lett. B 680, 4 (2009).
[77] C. Adloff et al. (H1 Collaboration), Phys. Lett. B 483, 23 (2000).

[78] S. J. Brodsky, E. Chudakov, P. Hoyer, and J. M. Laget, Phys. Lett. B 498, 23 (2001).

[79] E. Martynov, E. Predazzi, and A. Prokudin, Eur. Phys. J. C 26, 271 (2002).

[80] E. Martynov, E. Predazzi, and A. Prokudin, Phys. Rev. D 67, 074023 (2003).

[81] B. Gittelman, K. M. Hanson, D. Larson, E. Loh, A. Silverman, and G. Theodosiou, Phys. Rev. Lett. 35, 1616 (1975).

[82] J. M. Laget and R. Mendez-Galain, Nucl. Phys. A581, 397 (1995).

[83] A. Sibirtsev, S. Krewald, and A. W. Thomas, J. Phys. G 30, 1427 (2004).

[84] A. N. Hiller Blin, C. Fernández-Ramírez, A. Jackura, V. Mathieu, V. I. Mokeev, A. Pilloni, and A. P. Szczepaniak, Phys. Rev. D 94, 034002 (2016).

[85] H. Huang, C. Deng, J. Ping, and F. Wang, Eur. Phys. J. C 76, 624 (2016).

[86] H. Huang and J. Ping, Phys. Rev. D 99, 014010 (2019).

[87] Y.-H. Lin, C.-W. Shen, and B.-S. Zou, Nucl. Phys. A980, 21 (2018).

[88] G. Yang, J. Ping, and J. Segovia, Phys. Rev. D 99, 014035 (2019).

[89] C.-W. Shen, D. Rönchen, U.-G. Meißner, and B.-S. Zou, Chin. Phys. C 42, 023106 (2018).

[90] T. Gutsche and V.E. Lyubovitskij, Phys. Rev. D 100, 094031 (2019).

[91] M. Tanabashi et al. (Particle Data Group), Phys. Rev. D 98, 030001 (2018).

[92] J.-J. Wu, T.-S. H. Lee, and B.-S. Zou, Phys. Rev. C 100, 035206 (2019).

[93] A. Accardi et al., Eur. Phys. J. A 52, 268 (2016).

[94] D. Boer et al., arXiv:1108.1713.

[95] Y.-P. Xie, X. Cao, Y.-T. Liang, and X. Chen, arXiv: 2003.11729.

[96] Y. Huang, J. J. Xie, J. He, X. Chen, and H. F. Zhang, Chin. Phys. C 40, 124104 (2016).

[97] S. Lüders, A measurement of the beauty production cross section via $B \rightarrow J / \psi X$ at HERA, Ph.D. thesis, Swiss Federal Institute of Technology Zurich, 2001, https:// doi.org/10.3929/ethz-a-004280689.

[98] C. Adloff et al. (H1 Collaboration), Phys. Lett. B 467, 156 (1999); 518, 331(E) (2001).

[99] R. K. Ellis and P. Nason, Nucl. Phys. B312, 551 (1989).

[100] S. Frixione, M. L. Mangano, P. Nason, and G. Ridolfi, Phys. Lett. B 348, 633 (1995). 